\title{
A hybrid X-mode reflectometry first fringe detection algorithm for JET
}

\author{
P. J. Carvalho*仿, L. Meneses, J. Santos, B. Gonçalves and JET contributors \\ EUROfusion Consortium, JET, Culham Science Centre, Abingdon, OX14 3DB, UK \\ Instituto de Plasmas e Fusão Nuclear, Instituto Superior Técnico, Universidade de Lisboa \\ Av. Rovisco Pais, 1049-001 Lisboa, Portugal \\ E-mail: pedro.carvalho@ipfn.tecnico.ulisboa.pt
}

\begin{abstract}
At the Joint European Torus (JET), the presently implemented density profile by X-mode Frequency Modulated - Continuous Wave reflectometry system, known as KG10, is not living up to its full potential. This is due to an unreliable first fringe detection, and excessive filtering of the full signal which can result in profiles with little detail.

This paper aims to address the first of these problems, the first fringe detection. Since no documentation is available regarding the implementation currently in use, there are no assurances about how the present algorithm works, but a glance at successive profiles highlights its unreliable nature. Typically, first fringe detection algorithms used in X-mode reflectometry rely on detecting at which point the beat signal's power increases significantly.

The method introduced in this work uses the signal's power as the main discriminant, but also takes into consideration the spectral behavior of the signal in those instances where the power alone doesn't relay a consistent detection. Large deviations between consecutive sweeps of the reflectometer are prevented, thus yielding a stabler detection of the first fringe.

As an extra problem, JET's FM-CW system displays low power at the end of the Q-band and at the beginning of the V-band, where both bands overlap, between 51 and $53 \mathrm{GHz}$. This causes a lack of reliability on the power based methods, leading to an increased prevalence of the frequency based ones.

This hybrid power-frequency method yields promising results, providing a solid platform for subsequent relevant physics results.
\end{abstract}

1st EPS conference on Plasma Diagnostics

14-17 April 2015

Frascati, Italy

\footnotetext{
* Speaker.

${ }^{\dagger}$ Acknowledgments This work has been carried out within the framework of the EUROfusion Consortium and has received funding from the Euratom research and training programme 2014-2018 under grant agreement No 633053. IST activities also received financial support from "Fundação para a Ciência e Tecnologia" through project PestOE/SADG/LA0010/2013. The views and opinions expressed herein do not necessarily reflect those of the European Commission.

${ }^{*}$ See the Appendix of F. Romanelli et al., Proceedings of the 25th IAEA Fusion Energy Conference 2014, Saint Petersburg, Russia
} 


\section{Introduction}

Frequency Modulated - Continuous Wave (FM-CW) reflectometry is a common diagnostic for obtaining plasma density profiles in tokamaks [1, 2, 3, 4]. There are two types of density profile measurements: using O-mode and X-mode. At JET, only X-mode is currently used to determine density profiles. JET's FM-CW X-mode reflectometry system (KG10) is composed of 4 bands $(\mathrm{Q}, \mathrm{V}, \mathrm{W}$ and $\mathrm{D})$. Each of the bands performs a sweep over the probing frequencies in $10 \mu \mathrm{s}$ and can start a new sweep every $15 \mu \mathrm{s}$. Data is acquired at 200MSPS, yielding 2000 points per sweep. In this mode, the probed density layer has a dependency on the cyclotron frequency which places the first measured position within the typical reflectometry probing frequencies (40 $135 \mathrm{GHz}$ ). Assuming the first fringe corresponds to a location of zero plasma density, the position corresponding to this first detection can be inferred from the magnetic field profile.

The first fringe is determined by detecting the frequency for which the power of the beating of the launched and received waves goes above a particular threshold [4]. In general, this approach works well, but the existence of reflections in the wave-guides and an imperfect conduction of a few frequencies can lead to an unexpected wavy behavior of the power as a function of the probing frequency. Both these effects are present at JET's system. The algorithm currently calculating density profiles at JET is poorly documented, so the methodology employed to determine the first fringe is unknown to this author. It is known that the existing profiles are often unusable for physics studies, at least due to an unreliable detection of the position of the edge of the plasma.

This work aims to provide a novel methodology for the determination of the first fringe. It addresses the limitations imposed by a simple threshold on the signal's power by adding the frequency content of the signal as a second factor in that determination.

\section{First Fringe Algorithm}

The first step to determine which probing frequency corresponds to the first fringe is to select on which band to search for it. The probing frequency $\left(f_{p}\right)$ range corresponding to a potential first fringe in the range $3.75<R<4.0 \mathrm{~m}$ (from inside the separatrix to the limiter shadow) is determined by the magnetic field profile provided by the Flush library [6]. All subsequent analysis is performed within this range. If it falls in an overlap of two bands, the band for which a larger frequency interval is available gets selected. At JET, the intersection of the $\mathrm{Q}$ and $\mathrm{V}$ bands, $51<f_{p}<53 \mathrm{GHz}$, presents a particular challenge as both of those bands' signal is low in this region. The band with the largest search frequency range out of this untrustworthy region is selected.

For the selected band, the spectrogram of the raw signal is calculated, with a windowing of 64 points, zero-padded to 128 points, and a window step of 2 points, and the beat frequency and group delay $\tau_{g}$ are extracted as in [5]. Fig. 1 shows an example of a spectrogram for KG10's X-mode V band with the beat frequency detected as well as it's power.

Four different methods are then run to determine the first fringe.

1) Main Beat Frequency based detection (F0_G): Starting at the higher probing frequencies, where the beat frequency line is expected to be stable as it should be detecting the pedestal, and working towards the lower frequencies, search for a significant difference from the starting value. 


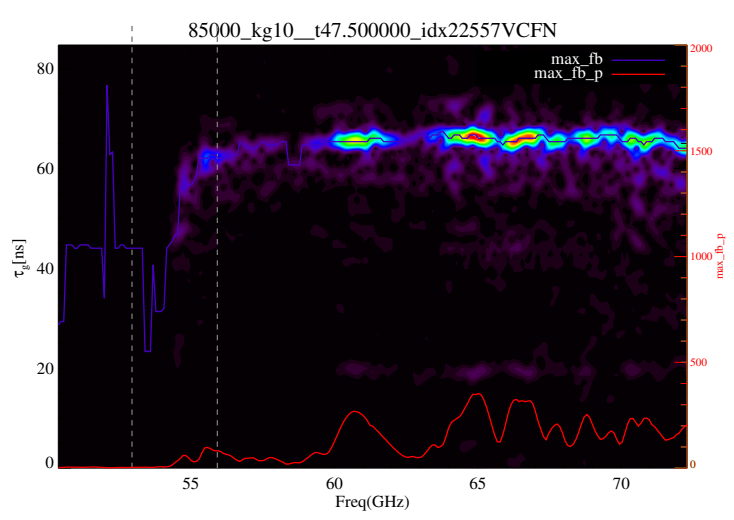

Figure 1: A spectrogram of the $\mathrm{V}$ band's raw signal. In purple, the group delay follows the maximum of the spectrogram's power (red). The vertical grey dashed lines border the location where the first fringe is expected to be, based on the magnetic field profile. The spectrogram is inverted so that a rise in $\tau_{g}$ typically accompanies a rise in signal power.

This threshold is half of the span from the minimum to the maximum group delay. The chosen $f_{p}$ is the first that remains below this value for, at least, $0.3 \mathrm{GHz}$.

2) Secondary Beat Frequency Based Detection (F0_C): This is the only method that depends on another. On the first sweep, it takes the same value as the main beat frequency algorithm (F0_G). On the other sweeps, a search is made for a point with a slope of the beat frequency line similar to the slope of the previous good sweep's line at the frequency detected, restricted to a distance of $0.2 \mathrm{GHz}$, from that previous. A good sweep is one that hasn't been marked as bad by the subsequent processing and there can be several such bad sweeps in sequence. If no suitable slope is detected within this range, it returns the same as the previous sweep and the usability flag is set to false.

3) Power Based Detection (F0_D): Heavily inspired by DIII-D's [4], it follows the power of the beat frequency line on the spectrogram (max_fb_p in Fig 1). Starting at the lowest $f_{p}$ the algorithm searches for a point which exceeds $18 \%$ of the maximum power plus the minimum power in the same range. The point is accepted if the next $0.3 \mathrm{GHz}$ are also above this threshold. If there's no point which satisfies this condition, then this algorithm returns the maximum frequency in the range under consideration.

4) Secondary Power Based Detection (F0_I): This method is very similar to the previous Power Based Detection, but it starts at the highest probing frequency and searches for a point which crosses the same threshold and remains below it for $0.3 \mathrm{GHz}$.

\subsection{Hybrid Fo}

This algorithm combines all the methods and selects the best candidate for first fringe, F0.

Any method with a detection close to the edge of the range where the first fringe is expected is first flagged as bad. The first sweep must be handled differently from the subsequent ones because some dependence on the previous sweep is included in the others. In the general case where the three methods are usable, if F0_D and F0_I are close $(<0.15 \mathrm{GHz})$, the lowest one of them is 
selected. If they are not close, F0_G is selected. If any one of the three methods is not usable, the lowest value of the two remaining is selected.

When F0 is sought on a different band from the previous sweep, it is processed as a first sweep, unless the thus determined F0 is farther than $0.6 \mathrm{GHz}$ from the previous good sweep. In this case, F0_C is selected, regardless of its usability flag, as it is naturally close to the previous F0.

The following description is valid for the remaining sweeps.

If the maximum power of the beat frequency component of the spectrogram lies below a threshold (17 for the V band, and 10 otherwise), the power based methods are not considered reliable. The first fringe is then given by one of the closest beat frequency based methods to the previous sweep's first fringe. However, if F0_C's non-usability flag is raised, the first fringe becomes F0_G. If not, a further check is employed: if the distance from F0_G to F0_C is greater than the distance from F0_G to F0_D and from F0_G to F0_I, then F0_G is definitely chosen.

If the power based methods are reliable, the following sequence is run:

1) If the power based methods, F0_D and F0_I, yield values close to each other $(<0.15 \mathrm{GHz})$, select the one closest to F0_G. 2) If the result from the previous case is farther from the previous sweep than F0_G and the signal power at F0_G is at least twice the power threshold used for F0_D and F0_I, or the distance from F0_G to F0_C is less than $0.13 \mathrm{GHz}$, then F0_G is selected. 3) If the result from the previous case is more than $0.45 \mathrm{GHz}$ away from the previous sweep's first fringe, select the method which yields the closest value to the previous sweep. 4) If the result from the previous case is still far from the previous sweep's first fringe, disregard the usability flags on all the 4 methods and select the method that yields the closest value to the previous sweep. The sweep is marked as bad. 5) When the power based methods, F0_D and F0_I, do not yield values close to each other, the true first fringe should be above the minimum and below the maximum of these two methods. Because of this disagreement, the frequency based methods are the only available and the one closest to the previous sweep's F0 is selected if both have their usability flag marked as good. If only one of them has a good usability flag, then that method is selected. If this selected value is more than $0.45 \mathrm{GHz}$ away from the previous sweep's F0, then, of all the four candidates, the closest is selected, constrained by the minimum and maximum values of the power based methods. If that result is still far from the previous, this previous is used and the result is marked as bad. After 6 consecutive bad sweeps, the median of all 4 methods and the previous good sweep is taken and is marked as good.

If none of the previous steps provides a result, then the maximum frequency from all the four methods is selected and it is marked as bad. This ensures that the first fringe is detected as far away from the wall as possible, in an area where some signal is really present and a subsequent density profile can be built free from a wrong signal at the edge, but probably with a wrong first position.

\section{Results and Comparisons}

In general, the strategies developed in this work return an accurate value for the first fringe.

In Fig. 2, the sceptrogram of the selected band is cropped to the region of the two dashed lines in Fig. 1. The max_fb line follows the maximum value of the FFTs in the spectrogram and is the one tracked by the frequency based methods. The max_fp_p line represents the power level of the FFT at the max_fb line and is the one tracked by the power based methods. The result from the 4 


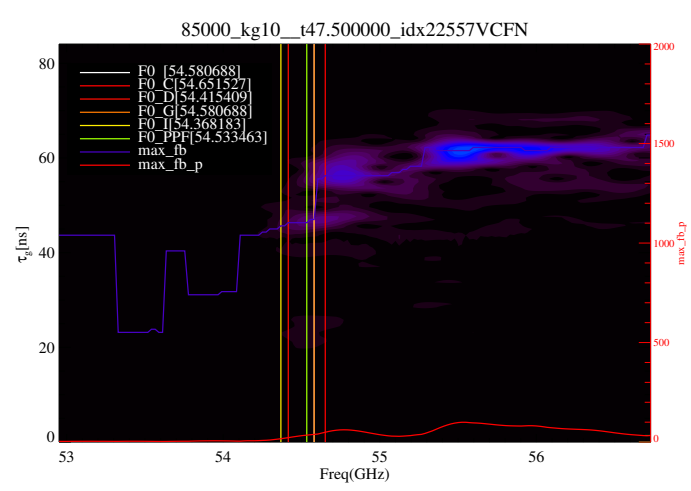

Figure 2: Spectrogram for the same sweep as in Fig.1 with the detected frequencies for each of the 4 methods presented. The selected first fringe (F0) is also shown.

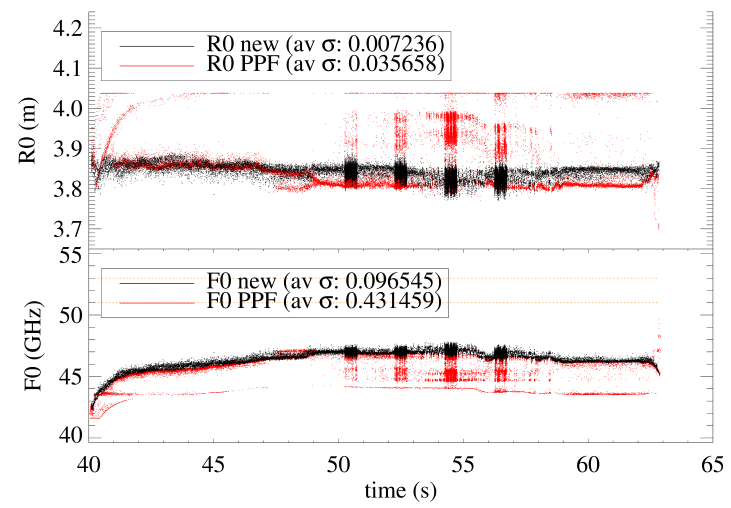

Figure 3: First Fringe (F0) and position of zero density (R0) calculated by the method outlined in this paper (new) and the currently implemented at JET (PPF) for the whole pulse \#85300.

methods (F0_C, F0_D, F0_G and F0_I) and the hybrid (F0) and the one calculated automatically by the currently implemented algorithm at JET (F0_PPF) are the vertical lines, with the value in the legend.

Fig. 2 shows a case where the power based methods were deemed less reliable than the frequency based ones. In this case, F0_G was selectd by the hybrid algorithm, because the power based methods yield a value a bit further away from the previous than F0_G. Analysing the max_fb_p line, the F0_I probing frequency marks the very start of the rise of this curve. Judging from the max_fb line, F0_G is correctly detected at the position of a suficient difference from the area with a constant max_fb at $55.5-56 \mathrm{GHz}$.

From single sweeps, it is not obvious how this hybrid method compares with the one currently implemented at JET. Fig. 3, on the bottom, displays the resulting F0 for this method (F0 new) and the automatic one (F0 PPF) for pulse \#85300. This pulse was selected to represent the problems found in "F0 PPF". This was a low magnetic field pulse which maintained the first fringe in the range of the Q-band. The top plot of the figure shows the corresponding radial position, obtained 
from the magnetic field profile which is supplied by the flush library [6]. On the legend, the "av $\sigma^{\prime \prime}$ is the average of a moving standard deviation for each of the quantities. This value quantifies the spreading obtained by each of the two algorithms which, for this pulse, is frankly less for "F0 new". For other pulses, where "F0 PPF" fails less, the "av $\sigma$ " is only marginally less.

The top plot of Fig. 3 shows the detected position of the first fringe at around $R=3.85 \mathrm{~m}$, which is the imposed location for the separatrix, thus lending credence to the result. The position yielded by "F0 PPF" does tend to go a bit further inward, but often moves outward reaching what appears to be an imposed maximum at $R=4.05 \mathrm{~m}$. The four bands of apparent increased dispersion of the detection in the range $50<t<57 \mathrm{~s}$ are windows of high sweeping rates $(30 \mu \mathrm{s}$ per sweep vs $1 \mathrm{~ms}$ ). In the region $47<t<49 \mathrm{~s}$, "F0 PPF" displays a secondary band, which "F0 new" doesn't.

During the majority of the pulse at a low sweeping rate, "FF0 PPF" presents a smaller dispersion than "FF0 new". However, analysing sequences of spectrograms, it has been found that "FF0 new", usually, seems to correctly follow the position where the beat frequency presents the start of a reflection at the scrape-off layer, while "FF0 PPF" seems sensitive to a small increase of the reflected power which, on some occasions, doesn't seem to correlate with the beat frequency.

\section{Discussion}

On this paper, a new algorithm for detection of the first fringe for the X-mode reflectometry diagnostic at JET is described. This algorithm relies on the behavior of both the power of the signal and the beat frequency, while preventing a significant deviation from one sweep to the next.

From the results presented in section 3, it is clear that the new algorithm manages to better keep track of the first fringe, yielding a more plausible initial position for the plasma. For most of the cases where the presently implemented algorithm at JET, "F0 PPF", fails, the new algorithm produces a better estimate for F0. These far outweigh the few occasions where "F0 PPF" provides a more accurate detection of the first fringe. There are also a few rare occasions where both algorithms fail to provide the correct first fringe. These are mostly due to a very distorted signal and are usually confined to single sweeps, perhaps due to the reflection of a turbulent structure at the edge of the plasma.

In general, this new algorithm presents an improvement over the currently installed one. It should then be possible to obtain a more reliable density profile from the KG10 diagnostic. The next iteration of this work is to provide a density profile fully based on the group delay obtained from the spectrogram of the signals from the various bands.

\section{References}

[1] M. Manso et al., Plas. Phys. Cont. Fus. 40 5, 747 (1998)

[2] Zhang Chongyang et al., Plas. Sci. Tech. 15 9, 857 (2013)

[3] W. L. Zhong et al., Rev. Sci. Instr. 851 (2014)

[4] G. Wang et al., Rev. Sci. Instr. 741525 (2003)

[5] J. Santos et al., IRW3 proceedings, 55 (1997)

[6] S. Pamela et al. CODAS - JET/EFDA, FLUSH User's Guide (2013) 